\title{
The concentration of erlotinib in the cerebrospinal fluid of patients with brain metastasis from non-small-cell lung cancer
}

\author{
YANMING DENG $^{1 *}$, WEINENG FENG $^{1 *}$, JING WU $^{2}$, ZECHENG CHEN $^{1}$, YICONG TANG $^{1}$, \\ HUA ZHANG $^{1}$, JIANMIAO LIANG ${ }^{1}$, HAIBING XIAN ${ }^{1}$ and SHUNDA ZHANG ${ }^{1}$ \\ ${ }^{1}$ Department of Head and Neck/Thoracic Medical Oncology, ${ }^{2}$ Oncology Institution, \\ The First People's Hospital of Foshan, Foshan, Guangdong 528000, P.R. China
}

Received February 5, 2013; Accepted July 19, 2013

DOI: $10.3892 / \mathrm{mco} .2013 .190$

\begin{abstract}
It has been demonstrated that erlotinib is effective in treating patients with brain metastasis from non-small-cell lung cancer. However, the number of studies determining the erlotinib concentration in these patients is limited. The purpose of this study was to measure the concentration of erlotinib in the cerebrospinal fluid of patients with brain metastasis from non-small-cell lung carcinoma. Six patients were treated with the standard recommended daily dose of erlotinib $(150 \mathrm{mg})$ for 4 weeks. All the patients had previously received chemotherapy, but no brain radiotherapy. At the end of the treatment period, blood plasma and cerebrospinal fluid samples were collected and the erlotinib concentration was determined by high-performance liquid chromatography-tandem mass spectrometry (HPLC-MS/MS). The average erlotinib concentration in the blood plasma and the cerebrospinal fluid was $717.7 \pm 459.7$ and $23.7 \pm 13.4 \mathrm{ng} / \mathrm{ml}$, respectively. The blood-brain barrier permeation rate of erlotinib was found to be $4.4 \pm 3.2 \%$. In patients with partial response (PR), stable disease (SD) and progressive disease (PD), the average concentrations of erlotinib in the cerebrospinal fluid were $35.5 \pm 19.0,19.1 \pm 8.7$ and $16.4 \pm 5.9 \mathrm{ng} / \mathrm{ml}$, respectively. In addition, the efficacy rate of erlotinib for metastatic brain lesions was $33.3 \%$, increasing to $50 \%$ in patients with $E G F R$ mutations. However, erlotinib appeared to be ineffective in cases with wild-type EGFR. In conclusion, a relatively high concentration of erlotinib was detected in the cerebrospinal fluid of patients with brain metastases from non-small-cell
\end{abstract}

Correspondence to: Dr Yanming Deng, Department of Head and Neck/Thoracic Medical Oncology, The First People's Hospital of Foshan, 81 Lingnan North Road, Foshan, Guangdong 528000, P.R. China

E-mail: yanmingdeng78@126.com

${ }^{*}$ Contributed equally

Key words: non-small-cell lung cancer, brain metastasis, erlotinib, cerebrospinal fluid lung cancer. Thus, erlotinib may be considered as a treatment option for this patient population.

\section{Introduction}

Approximately $10 \%$ of the patients with non-small-cell lung cancer are first diagnosed with brain metastases and 30-50\% develop brain metastasis during the course of the disease (1). Currently, whole-brain radiation therapy is the standard form of treatment for the majority of patients with brain metastases from non-small-cell lung cancer. In addition, a proportion of patients with a single metastasis may undergo operative treatment or stereotactic radiosurgery (1). Since the majority of chemotherapeutic agents are not able to pass through the blood-brain barrier, the efficacy of chemotherapy for non-small-cell lung cancer with brain metastases has not been well established (2). Overall, patients with brain metastases from non-small-cell lung cancer exhibit a poor prognosis, with a median survival of $\sim 6$ months following whole-brain radiotherapy (3).

Erlotinib is an epidermal growth factor receptor tyrosine kinase inhibitor (EGFR-TKI). Large randomized trials have demonstrated that erlotinib exerts a curative effect on advanced non-small-cell lung cancer, particularly in patients with EGFR-activating mutations (exon-19 deletion or exon-21 L858R mutation). The progression-free survival time may be as long as 14 months following erlotinib treatment (4,5). Recent studies demonstrated that erlotinib exerts a satisfactory curative effect on non-small-cell lung cancer with brain metastases and may still be effective in treating brain metastases after the failure of EGFR-TKI treatment (gefitinib) (6-9). Therefore, erlotinib is considered to reach adequately high concentrations in the cerebrospinal fluid (10).

A previous study on patients who underwent whole-brain radiotherapy demonstrated that this procedure may increase the permeability of the blood-brain barrier and eventually lead to a change in the erlotinib concentration in the cerebrospinal fluid (11). In this study, the plasma and cerebrospinal fluid concentration of erlotinib was measured in six patients with brain metastases from non-small-cell lung cancer who had not undergone whole-brain radiotherapy. Whether erlotinib is able to cross the blood-brain barrier to achieve an effective treatment concentration was also assessed. 
Table I. Patient characteristics.

\begin{tabular}{lcllclll}
\hline Cases & Age (years) & Gender & PS & Histology & EGFR & Smoking status & Previous Ctx cycles \\
\hline 1 & 57 & Female & 1 & Ad & Ex 19 deletion & Never & 2 \\
2 & 67 & Female & 1 & Ad & Ex 21 L858R & Never & 4 \\
3 & 62 & Male & 1 & Sq & Wild-type & Former & 6 \\
4 & 53 & Female & 0 & Ad & Ex 19 deletion & Never & Never \\
5 & 54 & Female & 1 & Ad & Wild-type & Never & 4 \\
6 & 59 & Female & 1 & Ad & Ex 19 deletion & \\
\hline
\end{tabular}

PS, performance status; EGFR, epidermal growth factor receptor gene; CTx, chemotherapy; Ad, adenocarcinoma; Sq, squamous cell carcinoma; Ex, exon; Never, never smoked; Former, former smoker.

\section{Patients and methods}

Patients. Six patients treated in our hospital between March, 2011 and March, 2012 were included in this study. The main inclusion criteria were as follows: age $\geq 18$ years, histologically confirmed non-small-cell lung cancer following failure of first- or second-line chemotherapy, asymptomatic brain metastases (no neurological symptoms or signs that could be easily controlled only with corticosteroids), measurable intracranial lesions, adequate bone marrow, liver and kidney function, and $>3$ months expected survival. The main exclusion criteria were as follows: simultaneous administration of chemotherapy or whole-brain radiotherapy and discontinuation or dose adjustment of erlotinib 1 week prior to collecting blood and cerebrospinal fluid samples.

This study was approved by the Ethics Committee of our hospital and each patient enrolled in the study provided informed consent.

Treatment and evaluation. The patients were treated with erlotinib (150 mg/day) until disease progression or occurrence of unacceptable toxicity. Chest computed tomography (CT) and brain magnetic resonance imaging (MRI) scans were performed to evaluate the curative effect after 4 weeks of treatment. Following the completion of the treatment cycle, chest CT and brain MRI scans were performed at 8-week intervals. If intracranial symptoms or neurological signs were aggravated, the curative effect of erlotinib was reassessed by chest $\mathrm{CT}$ and brain MRI. The overall curative effect was evaluated using Response Evaluation Criteria in Solid Tumors (RECIST 1.1). Standard effects included complete response (CR, disappearance of all target lesions), partial response (PR, at least $30 \%$ decrease in the sum of the longest diameter for all target lesions), progressive disease (PD, at least $20 \%$ increase in the sum of the longest diameter for all target lesions or appearance of new lesions) and stable disease (SD, the sum of the longest diameter for all target lesions is reduced, but less than PR, or increased, but less than PD). Progression-free survival was defined as the time from the beginning of erlotinib-based treatment to the appearance of PD.

Test methods. Blood and cerebrospinal fluid samples were collected from the six patients on the same day after 4 weeks of erlotinib treatment. According to a previous study (12), the plasma and cerebrospinal fluid concentration of erlotinib was measured by high-performance liquid chromatography-tandem mass spectrometry (HPLC-MS/MS). Primary lung tumor tissue samples from the six patients were also collected and paraffin-embedded. DNA was extracted from the tumor tissue and the mutation status of the EGFR gene was analyzed using the PCR technique to amplify and sequence EGFR exons 18-21.

Statistical analysis. Continuous variables were analyzed by the Student's t-test and the results are expressed as means \pm SD. Categorical variables were analyzed using the Fisher's exact test. A correlation analysis between the cerebrospinal fluid concentration of erlotinib and its curative effect was performed using analysis of variance. Survival analysis was conducted using the Kaplan-Meier method and $\mathrm{P} \leq 0.05$ was considered to indicate a statistically significant difference. All statistical analyses were performed by SPSS software version 13.0 (SPSS Inc., Chicago, IL, USA).

\section{Results}

Clinical characteristics. The clinical characteristics of the six patients are presented in Table I. The average age was $58.7 \pm 5.2$ years. The patient group comprised four females $(66.7 \%)$ and two males $(33.3 \%)$. Adenocarcinoma and non-smoking patients accounted for $83.3 \%(5 / 6)$. Four patients $(66.7 \%)$ harboured EGFR mutations. In addition, four patients had undergone first-line chemotherapy and two patients had received second-line chemotherapy.

Blood plasma and cerebrospinal fluid concentration, curative effect of erlotinib and location of tumor progression. The blood plasma and cerebrospinal fluid concentration, the curative effect of erlotinib and the site of tumor progression in the six patients are presented in Table II. The average concentration of erlotinib in the blood plasma and the cerebrospinal fluid was $717.7 \pm 459.7$ and $23.7 \pm 13.4 \mathrm{ng} / \mathrm{ml}$, respectively. The blood-brain barrier permeation rate of erlotinib (erlotinib concentration in the cerebrospinal fluid or blood plasma) was $4.4 \pm 3.2 \%$.

Of the six patients with brain metastasis, no patients exhibited a CR and two patients exhibited a PR. The effectiveness rate of erlotinib in the brain metastatic lesions was $33.3 \%(2 / 6)$. Of the four patients with EGFR mutations, two patients exhibited a PR. Thus, the effectiveness rate of erlotinib was $50 \%(2 / 4)$. 
Table II. Plasma concentrations of erlotinib, penetration rate of erlotinib and site of disease progression.

\begin{tabular}{|c|c|c|c|c|c|c|}
\hline Cases & $\begin{array}{l}\text { Plasma concentration } \\
\qquad(\mathrm{ng} / \mathrm{ml})\end{array}$ & $\begin{array}{l}\text { CSF concentration } \\
(\mathrm{ng} / \mathrm{ml})\end{array}$ & $\begin{array}{l}\text { Penetration } \\
\text { rate }(\%)\end{array}$ & $\begin{array}{l}\text { Brain metastases } \\
\text { response }\end{array}$ & $\begin{array}{c}\text { PFS } \\
\text { (months) }\end{array}$ & $\begin{array}{c}\text { Site of } \\
\text { progression }\end{array}$ \\
\hline 1 & 665 & 22.1 & 3.3 & PR & 9.2 & Brain \\
\hline 2 & 468 & 48.9 & 10.4 & PR & 10.2 & Bone \\
\hline 3 & 1230 & 20.6 & 1.7 & PD & 1.0 & Brain, lung \\
\hline 4 & 351 & 12.2 & 3.5 & PD & 1.0 & Brain \\
\hline 5 & 236 & 12.9 & 5.5 & SD & 2.8 & Brain, lung \\
\hline 6 & 1310 & 25.2 & 1.9 & $\mathrm{SD}$ & 5.6 & Brain, lung \\
\hline Mean \pm SD & $717.7 \pm 459.7$ & $23.7 \pm 13.4$ & $4.4 \pm 3.2$ & & & \\
\hline
\end{tabular}

CSF, cerebrospinal fluid; PFS, progression-free survival; PR, partial response; SD, stable disease; PD, progressive disease; SD, standard deviation.

Table III. Association between CSF concentration and response of brain metastases.

\begin{tabular}{lccc}
\hline Response & $\begin{array}{c}\text { Patient } \\
\text { no. }\end{array}$ & $\begin{array}{c}\text { CSF } \\
\text { concentration } \\
(\mathrm{ng} / \mathrm{ml})\end{array}$ & P-value \\
\hline PR & 2 & $35.5 \pm 19.0$ & 0.378 \\
SD & 2 & $19.1 \pm 8.7$ & \\
PD & 2 & $16.4 \pm 5.9$ & \\
\hline
\end{tabular}

CSF, cerebrospinal fluid; PR, partial response; SD, stable disease; $\mathrm{PD}$, progressive disease.

No PR or CR was observed in patients with wild-type EGFR. The median progression-free survival rate (MPFS) of the six patients was 2.8 months. The MPFS of patients with EGFR mutations was 5.6 months, whereas it was only 1 month in patients with the wild-type EGFR. Of the six patients investigated, five (83.33\%) exhibited PD of the brain lesions. Of these five patients, two $(33.3 \%)$ had PD of intracerebral brain lesions, whereas three patients had PD of intra- and extracerebral brain lesions.

Association between the concentration of erlotinib in the cerebrospinal fluid and curative effect. The association between the erlotinib concentration in the cerebrospinal fluid and the overall curative effect is presented in Table III. There was no significant difference $(\mathrm{P}=0.378)$ in the average concentration of erlotinib in the cerebrospinal fluid among the PR, SD and PD groups, indicating that there is no significant association between the concentration of erlotinib in the cerebrospinal fluid and the curative effect.

\section{Discussion}

The blood-brain barrier is composed of microvascular endothelial cells, pericytes and perivascular astrocytic end-feet. It closely connects the surrounding cells and inhibits drugs from entering the cerebrospinal fluid via blood circulation (13). However, when brain parenchymal metastases grow to $>1-2 \mathrm{~mm}$ in diameter, the structure and function of the blood-brain barrier is disrupted, allowing drugs to enter the brain parenchyma (14). Weber et al (15) used the ${ }^{11} \mathrm{C}$-marked erlotinib as a tracer with PET/CT to monitor a patient with brain metastasis from non-small-cell lung cancer. The results revealed that a high concentration of ${ }^{11} \mathrm{C}$-marked erlotinib was detected in the patient's cerebellar metastasis, whereas no ${ }^{11} \mathrm{C}$-marked erlotinib was detected in normal brain tissue. That study demonstrated that erlotinib is able to pass through the blood-brain barrier and accumulate in metastatic brain lesions. Recently, other studies $(16,17)$ have demonstrated that when a standard dose of erlotinib (150 mg/day) was used to treat patients with brain metastases from non-small-cell lung cancer, the cerebrospinal fluid concentration of erlotinib was increased to $28.7-54 \mathrm{ng} / \mathrm{ml}$ and the blood-brain barrier permeation rate was $2.77-5.1 \%$. The cerebrospinal fluid concentration of erlotinib was associated with its plasma concentration $(16,17)$. However, the number of similar studies is limited. Furthermore, since whole-brain radiotherapy may accelerate the opening of the blood-brain barrier and thus affect the erlotinib concentration in the cerebrospinal fluid, it is necessary to investigate the cerebrospinal fluid concentration of erlotinib in patients with brain metastases from non-small-cell lung cancer that have not undergone whole-brain radiotherapy prior to the drug administration. In this study, none of the patients had undergone whole-brain radiotherapy and the blood brain barrier permeability of erlotinib was found to be $4.4 \%$. The erlotinib concentration in the cerebrospinal fluid was $24 \mathrm{ng} / \mathrm{ml}$, which was higher compared to the $7.9 \mathrm{ng} / \mathrm{ml}$ median inhibitory concentration $\left(\mathrm{IC}_{50}\right)$ of the EGFR wild-type cell. This finding suggests that the standard dose of erlotinib for patients with brain metastases from non-small-cell lung cancer exerted a curative effect. However, similar to the type of micromolecular EGFR-TKI, the gefitinib concentration in the cerebrospinal fluid was distinctly less than its $\mathrm{IC}_{50}$ value and the erlotinib concentration (16-18). This is mainly due to the fact that the plasma peak concentration of gefitinib with the standard dose was $\sim 14 \%(1 / 7)$ that of erlotinib $(19,20)$ and the blood-brain barrier permeability of gefitinib was $\sim 1 \%$, which was significantly lower compared to that of erlotinib (16). This finding may explain the fact that erlotinib and not gefitinib was effective in treating patients with brain metastases.

The number of studies that have investigated the curative effect of erlotinib on non-small-cell lung cancer with 
brain metastases is limited. The efficacy rate of erlotinib in non-selected patients with brain metastases has been found to be $10-56 \%(6,7)$. However, in patients with EGFR mutations, the rate was increased to $70-80 \%$. The rate was found to be higher in patients with wild-type $\operatorname{EGFR}(8,9)$. Those results were consistent with the curative effect of erlotinib treating extracranial lesions in patients with EGFR mutations and non-small-cell lung cancer (5). In this study, we observed a PR in two of four patients. No curative effect was observed in patients with wild-type $E G F R$, which indicated that the $E G F R$ mutation is a strong predictor of the curative effect of erlotinib in metastatic brain lesions and extracranial lesions.

A previous study by Clarke et al suggested that an increase in the erlotinib dose likely improves the cerebrospinal fluid concentration, further increasing the efficacy of the treatment of brain metastases (21). That study reported that one patient with brain metastases from non-small-cell lung cancer and EGFR mutation exhibited remission of the brain metastatic lesions following treatment with a high dose of erlotinib $(1,500 \mathrm{mg} /$ day) instead of the standard dose, which exerted no curative effect. In this study, the concentration of erlotinib in the cerebrospinal fluid was not correlated with its curative effect. This may be due to the fact that the concentration of erlotinib in the cerebrospinal fluid of the six patients exceeded the therapeutic concentration in our study but was lower compared to the therapeutic concentration in the Clarke et al study (21). Thus, in patients with brain metastases from non-small-cell lung cancer and EGFR mutations it is important to determine the concentration of erlotinib in the cerebrospinal fluid when treatment with the standard dose of erlotinib has been unsuccessful. The curative effect of erlotinib may be enhanced by increasing the dose when the standard therapeutic dose achieves no improvement.

Whole-brain radiotherapy is the standard treatment for the majority of patients with brain metastases from non-small-cell lung cancer. However, simple whole-brain radiation therapy may not be the optimal choice, since the median survival rate is currently $\sim 6$ months. Recent studies $(8,9)$ have demonstrated that EGFR-TKI treatment of non-small-cell lung cancer with brain metastases yielded better results, particularly for patients with EGFR mutations. For this group of patients, the median survival rate was reported to be 12-16 months. Thus, this type of treatment may be an effective option for non-small-cell lung cancer with brain metastases $(8,9)$. However, the main failure pattern of this type of treatment is in the progression of intracranial lesions. Wu et al (7) reported that $90 \%$ of patients with brain metastases from non-small-cell lung cancer who had received second-line treatment with erlotinib exhibited intracranial lesion progression during the course of the disease. Moreover, Park et al (9) reported that in patients with non-small-cell lung cancer with EGFR mutations and brain metastases who had been treated with EGFR-TKIs, $81 \%$ of the failure pattern included intracranial lesion progression and $62 \%$ of the patients only exhibited intracranial lesion progression. In our study, five of the six patients $(83.3 \%)$ exhibited intracranial lesion progression, with only intracranial lesion progression in two patients (33.3\%). This result was consistent with the results of the studies mentioned above. Of note, when using EGFR-TKIs to treat patients with brain metastases from non-small-cell lung cancer, it is essential to enhance the long-term control of intracranial lesion progression. Lind et al (22) used erlotinib combined with whole-brain radiotherapy to treat seven patients with brain metastases from non-small-cell lung cancer. One of the seven subjects exhibited intracranial lesion progression, whereas the remaining six patients exhibited extracranial lesion progression. Those results suggested that metastatic brain lesions may be more effectively controlled when treated with erlotinib combined with whole-brain radiotherapy. The study by Park et al (9) demonstrated that the intracranial lesions in 14 patients were effectively controlled by radiotherapy following intracranial lesion progression after EGFR-TKI therapy. The median survival time without local treatment was prolonged to $\sim 13$ months. The above-mentioned studies also suggested that treatment with EGFR-TKIs or whole-brain radiotherapy alone failed to achieve the optimal effect. In the future, it is recommended that the two methods are combined to treat non-small-cell lung cancer patients with brain metastases. However, the optimal combination of the two types of treatment may achieve optimal results requires further studies, including a larger patient sample.

In conclusion, this study has demonstrated that a concentration of erlotinib in the cerebrospinal fluid exerting a curative effect may be achieved in patients with brain metastases from non-small-cell lung cancer, even when treated with the standard dose of erlotinib. Erlotinib exerts a curative effect on metastatic brain lesions and may be successfully used in patients with brain metastases from non-small-cell lung cancer. However, due to the small sample size included this study, the results require confirmation by further investigations in order to optimize the treatment of patients with brain metastases from non-small-cell lung cancer using the combination of erlotinib and whole-brain radiotherapy.

\section{Acknowledgements}

This study was supported by grants from the Wu Jieping Medical Foundation (no. 320.6750.11058).

\section{References}

1. Yamanaka R: Medical management of brain metastases from lung cancer (Review). Oncol Rep 22: 1269-1276, 2009.

2. Mehta MP, Paleologos NA, Mikkelsen T, et al: The role of chemotherapy in the management of newly diagnosed brain metastases: a systematic review and evidence-based clinical practice guideline. J Neurooncol 96: 71-83, 2010.

3. Gaspar LE, Mehta MP, Patchell RA, et al: The role of whole brain radiation therapy in the management of newly diagnosed brain metastases: a systematic review and evidence-based clinical practice guideline. J Neurooncol 96: 17-32, 2010.

4. Shepherd FA, Rodrigues Pereira J, Ciuleanu T, et al: Erlotinib in previously treated non-small-cell lung cancer. N Engl J Med 353: $123-132,2005$

5. Rosell R, Moran T, Queralt C, Porta R, et al: Screening for epidermal growth factor receptor mutations in lung cancer. N Engl J Med 361: 958-967, 2009.

6. Bai $\mathrm{H}$ and Han B: The effectiveness of erlotinib against brain metastases in non-small-cell lung cancer patients. Am J Clin Oncol 36: 110-115 2013

7. Wu YL, Zhou C, Cheng Y, et al: A phase II study (CTONG0803) of erlotinib as second-line treatment in advanced non-small cell lung cancer (NSCLC) patients (pts) with asymptomatic brain metastases (BM) after first-line chemotherapy (CT). J Clin Oncol 29 (Suppl 15): abs. 7605, 2011.

8. Porta R, Sánchez-Torres JM, Paz-Ares L, et al: Brain metastases from lung cancer responding to erlotinib: the importance of EGFR mutation. Eur Respir J 37: 624-631, 2011. 
9. Park SJ, Kim HT and Lee DH: Efficacy of epidermal growth factor receptor tyrosine kinase inhibitors for brain metastasis in non-small cell lung cancer patients harboring either exon 19 or 21 mutation. Lung Cancer 77: 556-560, 2012.

10. Masuda T, Hattori N, Hamada A, et al: Erlotinib efficacy and cerebrospinal fluid concentration in patients with lung adenocarcinoma developing leptomeningeal metastases during gefitinib therapy. Cancer Chemother Pharmacol 67: 1465-1469, 2011.

11. Wilson CM, Gaber MW, Sabek OM, et al: Radiation-induced astrogliosis and blood-brain barrier damage can be abrogated using anti-TNF treatment. Int J Radiat Oncol Biol Phys 74 934-941, 2009.

12. Zhang W, Siu LL, Moore MJ and Chen EX: Simultaneous determination of OSI-774 and its major metabolite OSI-420 in human plasma by using HPLC with UV detection. J Chromatogr B Analyt Technol Biomed Life Sci 814: 143-147, 2005.

13. Abbott NJ, Rönnbäck L and Hansson E: Astrocyte-endothelial interactions at the blood-brain barrier. Nat Rev Neurosci 7: 41-53, 2006.

14. Deeken JF and Loscher W: The blood-brain barrier and cancer: transporters, treatment, and Trojan horses. Clin Cancer Res 13 $1663-1674,2007$.

15. Weber B, Winterdahl M, Memon A, et al: Erlotinib accumulation in brain metastases from non-small cell lung cancer: visualization by positron emission tomography in a patient harboring a mutation in the epidermal growth factor receptor. $J$ Thorac Oncol 6: 1287-1289, 2011.
16. Togashi Y, Masago K, Masuda S, et al: Cerebrospinal fluid concentration of gefitinib and erlotinib in patients with non-small cell lung cancer. Cancer Chemother Pharmacol 70: 399-405, 2012.

17. Togashi Y, Masago K, Fukudo M, et al: Cerebrospinal fluid concentration of erlotinib and its active metabolite OSI-420 in patients with central nervous system metastases of non-small cell lung cancer. J Thorac Oncol 5: 950-955, 2010.

18. Zhao J, Chen M, Zhong W, Zhang L, et al: Cerebrospinal fluid concentrations of gefitinib in patients with lung adenocarcinoma. Clin Lung Cancer 14: 188-193, 2012.

19. Hidalgo M, Siu LL, Nemunaitis J, et al: Phase I and pharmacologic study of OSI-774, an epidermal growth factor receptor tyrosine kinase inhibitor, in patients with advanced solid malignancies. J Clin Oncol 19: 3267-3279, 2001.

20. Ranson M, Hammond LA, Ferry D, et al: ZD1839, a selective oral epidermal growth factor receptor-tyrosine kinase inhibitor, is well tolerated and active in patients with solid, malignant tumors: results of a phase I trial. J Clin Oncol 20: 2240-2250, 2002.

21. Clarke JL, Pao W, Wu N, et al: High dose weekly erlotinib achieves therapeutic concentrations in CSF and is effective in leptomeningeal metastases from epidermal growth factor receptor mutant lung cancer. J Neurooncol 99: 283-286, 2010.

22. Lind JS, Lagerwaard FJ, Smit EF, et al: Phase I study of concurrent whole brain radiotherapy and erlotinib for multiple brain metastases from non-small-cell lung cancer. Int J Radiat Oncol Biol Phys 74: 1391-1396, 2009. 